\title{
Intracellular Acidification Reversibly Reduces Endocytosis at the Neuromuscular Junction
}

\author{
Clark A. Lindgren, ${ }^{2}$ Dennis G. Emery, ${ }^{1}$ and Philip G. Haydon ${ }^{1}$ \\ ${ }^{1}$ Laboratory of Cellular Signaling, Department of Zoology and Genetics, lowa State University, Ames, lowa 50011, and \\ 2Department of Biology, Grinnell College, Grinnell, lowa 50112
}

\begin{abstract}
The close spatial and temporal coupling of endocytosis and exocytosis in nerve terminals has made it difficult to elucidate the mechanisms and the regulation of endocytosis per se. Despite significant advances in our knowledge of the molecules involved in endocytosis, it has not yet been possible to selectively manipulate endocytosis in nerve terminals. We report that the substitution of propionate for chloride in the saline bathing a lizard neuromuscular junction reduces internal $\mathrm{pH}$ and reversibly blocks activity-dependent endocytosis. When intraterminal $\mathrm{pH}$ is reduced by $\sim 0.7 \mathrm{pH}$ units, the uptake of FM1-43 in nerve
\end{abstract}

terminals, but not activity-dependent destaining, is reduced. Normalization of intracellular $\mathrm{pH}$ by removing the propionate, raising extracellular $\mathrm{pH}$, or adding ammonium chloride immediately restores FM1-43 uptake. Electron microscopy indicates that intracellular acidification reversibly reduces activitydependent endocytosis in nerve terminals, because depolarization in propionate saline leads to a depletion of vesicles and the appearance of large intramembraneous infoldings.

Key words: exocytosis; endocytosis; FM1-43; synaptic vesicle; neuromuscular junction
In various non-neuronal cells, experimental manipulations that reduce intracellular $\mathrm{pH}$ have been shown to block endocytosis (Davoust et al., 1987; Sandvig et al., 1987; Heuser, 1989). In cultured chick fibroblasts, cytoplasmic acidification has been shown to arrest the endocytotic uptake of HRP without decreasing the number of clathrin lattices (Heuser, 1989). This suggests that internal acidification acts at a later step in endocytosis than the formation of clathrin lattices. Substitution of chloride with propionate in the external saline blocks vesicle recycling at the frog neuromuscular junction (Gennaro et al., 1978; Florey and Kriebel, 1988; Ceccarelli et al., 1989). Ceccarelli et al. concluded that external chloride is required for the recycling of synaptic vesicles. However, because propionate is the anion of a weak acid (propionic acid), which is known to acidify the interior of cells when present in the external saline (Sharp and Thomas, 1981), another possible conclusion is that vesicle recycling at the neuromuscular junction is also sensitive to a decrease in intracellular $\mathrm{pH}$. Dynamin, a GTPase critically required for vesicle endocytosis (Chen et al., 1991; van der Bliek and Meyerowitz, 1991), forms a pH-sensitive macromolecular complex with clathrin adapter protein AP2 (Wang et al., 1995) and is believed to act in the fission stage of endocytosis, subsequent to the formation of the clathrin lattice (see De Camilli and Takei, 1996; Takei et al., 1995, 1996).

The $\mathrm{pH}$ sensitivity of the molecular association between dynamin and AP2, the block of endocytosis during acidification in non-neuronal cells, and the inhibition of vesicle recycling in frog neuromuscular junctions by external propionate all raise the possibility that endocytosis in nerve terminals may be manipulated

Received Nov. 21, 1996; revised Feb. 14, 1997; accepted Feb. 18, 1997.

This work was supported by National Institutes of Health Grants NS26650 and NS24233, a McKnight Foundation Fellowship to P.G.H., and a Harris Faculty Fellowship to C.A.L. We thank Drs. Sheldon Shen and Louis-Eric Trudeau for discussions during the course of this work and for critically reading a version of this manuscript.

Correspondence should be addressed to Dr. Philip G. Haydon, Laboratory of Cellular Signaling, Room 339 Science II, Iowa State University, Ames, IA 50011.

Copyright (C) 1997 Society for Neuroscience $\quad 0270-6474 / 97 / 173074-11 \$ 05.00 / 0$ experimentally by changing internal $\mathrm{pH}$. To test this possibility, we studied the $\mathrm{pH}$-dependence of endocytosis in motor nerve terminals of the ceratomandibularis muscle of the lizard (Anolis carolinensis). The large, superficial presynaptic boutons and thin layer of muscle cells (only one to three cells thick) provide ideal optical conditions for using the $\mathrm{pH}$ indicator BCECF and the styryl dye FM1-43 to monitor intracellular $\mathrm{pH}$ and endocytosis, respectively, in a presynaptic terminal.

We demonstrate that replacement of chloride with propionate in the external saline acidifies lizard neuromuscular junctions. Experimentally induced reductions of intraterminal $\mathrm{pH}$ from $\sim 7.5$ to 6.8 reversibly reduce activity-dependent uptake of FM1-43 without impairing exocytosis. Electron microscopy revealed that endocytosis in lizard motor nerve terminals is blocked by intracellular acidification, because terminals were depleted of synaptic vesicles when stimulated in the presence of external propionate.

Some of these results have been reported in preliminary form (Lindgren et al., 1996).

\section{MATERIALS AND METHODS}

Preparation. Small $(5-8 \mathrm{~cm})$ lizards, Anolis carolinensis, were obtained from Carolina Biological Supply Company (Burlington, NC). Before experimentation, the brains of the lizards were destroyed with a pithing needle (Lindgren and Moore, 1989). To minimize discomfort, the lizards were placed in a container at $5^{\circ} \mathrm{C}$ for at least $10 \mathrm{~min}$ before decerebration. The cold anesthesia was verified by the absence of a tail prick or corneal reflex. The ceratomandibularis muscle and its motor nerve, a small branch of the hypoglossal nerve, were then dissected from the lower jaw and perfused with saline of the following composition (in $\mathrm{mM}$ ): $\mathrm{NaCl} 158$; $\mathrm{KCl} 2 ; \mathrm{MgCl}_{2} 2 ; \mathrm{CaCl}_{2} 2$; glucose 10; HEPES 5 .

Staining and destaining with FM1-43. Nerve-muscle preparations were secured in a custom-made plexiglass chamber that contained \#1 coverslip glass on the bottom. The motor nerve terminals were stained by bathing them in saline containing $2 \mu \mathrm{M}$ FM1-43 (Molecular Probes, Eugene, OR). The nerve terminals were stimulated either by applying pulses of current to the motor nerve through a suction electrode or by bathing the preparation in a saline containing a high concentration of potassium ions (high-K saline). This high-K saline was composed of (in $\mathrm{mM}$ ): $\mathrm{NaCl} 100$; $\mathrm{KCl} 60 ; \mathrm{MgCl}_{2} 2 ; \mathrm{CaCl}_{2}$ 2; glucose 10; HEPES 5. When it was necessary to block $\mathrm{Ca}^{2+}$ influx, the saline composition was (in $\mathrm{mM}$ ): $\mathrm{NaCl} 100 ; \mathrm{KCl}$ 


\section{LOADING}
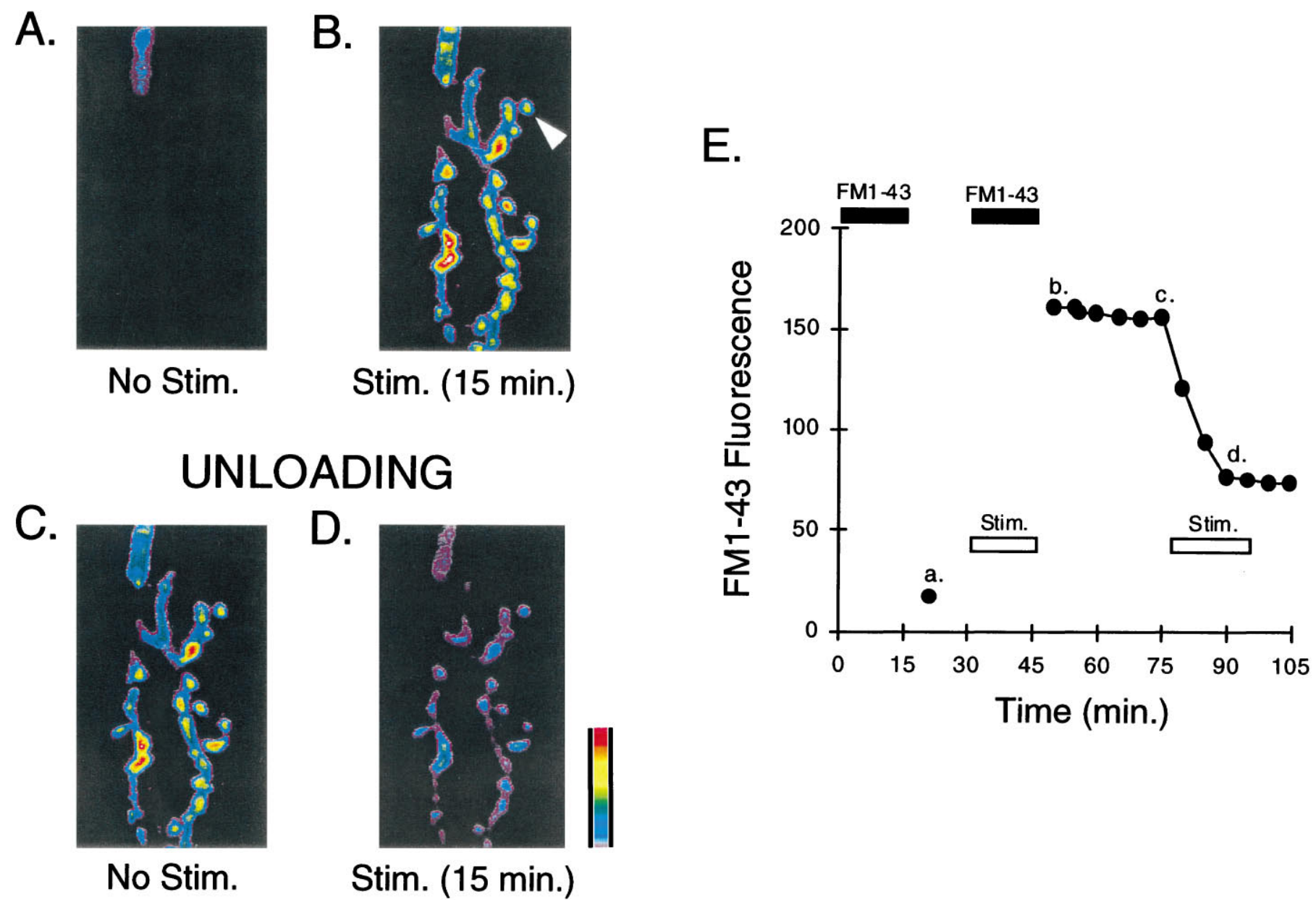

Figure 1. Staining and destaining of lizard motor nerve terminals with FM1-43 requires stimulation of the motor nerve. $A-D$, The intensity of FM1-43 fluorescence is depicted by pseudocolor images created from digitized data acquired by a confocal microscope. The images were aligned and identically stretched for maximal contrast. $A$, FM1-43 $(2 \mu \mathrm{M})$ was applied to a preparation for $15 \mathrm{~min}$; the preparation was then washed for 5 min in control saline. Note that the only structure to retain FM1-43 was the motor nerve's myelin sheath, seen at the top of the image. $B$, The same preparation was reexposed to $2 \mu \mathrm{M}$ FM1-43 for $15 \mathrm{~min}$ while stimulating the motor nerve; stimulation was then stopped, and the preparation was washed for $5 \mathrm{~min}$ in control saline. Under these conditions (i.e., stimulation), the boutons and branches of the nerve terminal acquired the FM1- 43 stain. $C$, The preparation was then washed for $25 \mathrm{~min}$ in control saline. During this time, only minimal FM1-43 was lost. $D$, While continuing to wash the preparation, the motor nerve was again stimulated. After $15 \mathrm{~min}$, the fluorescence intensity had decreased considerably throughout the nerve terminal. $E$, The same result depicted in $A-D$ is shown graphically. The average intensity over one of the boutons (arrowhead in $B$ ) was quantified, background-subtracted, and plotted as a function of time. $a-d$ Indicate which data points correspond to the panels of pseudocolor images.

60; $\mathrm{MgCl}_{2} 3 ; \mathrm{CdCl}_{2}$ 1; glucose 10; HEPES 5. When propionate and/or ammonium chloride were added to the saline, the concentration of $\mathrm{NaCl}$ was reduced by an equimolar amount. Unless stated otherwise, the $\mathrm{pH}$ of the saline was 7.3. Tetrodotoxin ( $1 \mu \mathrm{M}$; RBI, Natick, MA) was present in all experiments except that summarized in Figure 1.

Fluorescence microscopy and image processing. Nerve-muscle preparations were viewed with a Noran Odyssey laser scanning confocal microscope. Preparations stained with FM1-43 were viewed through a Nikon $60 \times 1.4$ NA oil immersion lens. The confocal slit was set at a width of 15 $\mu \mathrm{m}$. The preparation was excited with light of $488 \mathrm{~nm}$ wavelength, and light emitted at $>515 \mathrm{~nm}$ was collected and digitized using a matrox card and Image- 1 software (Universal Imaging, West Chester, PA). At 5 or 10 min intervals, 32 images were collected and averaged. The average light intensity emitted from five to six brightly stained boutons selected from a single nerve terminal was calculated at each time interval, and this average intensity was monitored as a function of time.

Measuring $\mathrm{pH}$ changes with $B C E C F$. Preparations were exposed to an external bathing solution containing $10 \mu \mathrm{M}$ of the membrane permeant acetoxymethyl ester of BCECF (BCECF-AM, Molecular Probes) for 60 $\mathrm{min}$. The preparations were then washed in control saline for 30-60 min to allow deesterification of the BCECF-AM that had diffused into the terminals. Changes in $\mathrm{pH}$ were monitored by measuring the fluorescence of the BCECF trapped in the nerve terminals (excitation, $488 \mathrm{~nm}$; emission, $>515 \mathrm{~nm}$ ). Because BCECF was also trapped in the muscle cells by this procedure, measurements were made only if the confocal microscope could clearly resolve the fluorescence in the terminal from the fluorescence in the muscle. Most often, these terminals were located on the sides of muscle fibers (as viewed from above), separated by at least $10-20 \mu \mathrm{m}$ from neighboring fibers. Because confocal microscopy was required to resolve nerve terminals, we were unable to perform ratiometric imaging, because the laser does not have the necessary line at $440 \mathrm{~nm}$. Therefore, all values are reported as $\Delta F / F_{\mathrm{o}}$.

Estimating normal intracellular $\mathrm{pH}$. To estimate normal intracellular $\mathrm{pH}$, lizard motor nerve terminals were loaded with BCECF (via the AM ester), and their cell membranes were made permeable to hydrogen and potassium ions by adding $25 \mu \mathrm{M}$ of the ionophore nigericin. At the same time that nigericin was applied, the concentration of potassium in the external saline was increased to $160 \mathrm{~mm}$ to equalize internal and external potassium concentrations. Because this treatment makes the nerve terminal membrane freely permeable to hydrogen ions and also dissipates any membrane potential, hydrogen ions rapidly diffuse down their concentration gradient until the internal $\mathrm{pH}$ is equal to the external $\mathrm{pH}$. 
Because the nerve terminals are loaded with BCECF, the movement of hydrogen ions across the membrane can be detected as a change in fluorescence. If the net movement of hydrogen ions is into the terminal, BCECF fluorescence will decrease, whereas if the net movement is outward, BCECF fluorescence will increase. In the null-point method, the entire procedure is repeated systematically, each time setting the external $\mathrm{pH}$ to a different value. The external $\mathrm{pH}$ at which the addition of nigericin does not change BCECF fluorescence is equal to the normal internal $\mathrm{pH}$.

Note that this estimate of intracellular $\mathrm{pH}$ may not reflect the actual cytoplasmic $\mathrm{pH}$, but rather may reflect an average of all intracellular compartments. If the nigericin accessed any of the intracellular membranes, such as the mitochondria or synaptic vesicles, the cytosolic $\mathrm{pH}$ may have changed before the equilibration of hydrogen ions across the cell membrane. However, in performing the "null-point" measurements, the response to the application of nigericin was always rapid and never showed indications of multiple time constants, decreasing the likelihood that much intracellular reequilibration occurred.

Electron microscopy. Nerve-muscle preparations were fixed for $2 \mathrm{hr}$ at room temperature with a mixture of $2 \%$ glutaraldehyde, $1 \%$ paraformaldehyde, and $0.25 \%$ tannic acid in either normal saline or $100 \mathrm{~mm}$ propionate saline (i.e., normal lizard saline in which $100 \mathrm{~mm}$ chloride was replaced with propionate). This was followed by two rinses in $100 \mathrm{~mm}$ propionate saline and fixation for $30-60 \mathrm{~min}$ with $0.5 \% \mathrm{OsO}_{4}$ and $0.8 \%$ $\mathrm{K}_{3} \mathrm{Fe}(\mathrm{CN})_{6}$ in $100 \mathrm{~mm}$ propionate saline. The tissue was rinsed with water, stained en block for $2 \mathrm{hr}$ with $1 \%$ aqueous magnesium uranyl acetate, and embedded in epon-araldite resin after ethanol-propylene oxide dehydration. Thin sections were stained with lead citrate before viewing.

\section{RESULTS}

\section{Staining and destaining lizard nerve terminals with FM1-43}

We first determined that FM1-43 stains lizard motor nerve terminals under conditions similar to those reported previously for frog, mouse, and rat muscles (Betz and Bewick, 1992; Betz et al., 1992; Ribchester et al., 1994). As shown in Figure 1, the application of $2 \mu \mathrm{M}$ FM1-43 to a lizard ceratomandibularis muscle for 15 min followed by a 5 min wash with control saline results in only low levels of background staining (Fig. 1A). Other than in the myelin sheath covering the motor nerve, fluorescence was only slightly above background (Fig. $1 E$, data point $a$ ). In contrast, if FM1-43 was applied while the nerve was being stimulated (15 min of continuous stimulation at $1 \mathrm{~Hz}$ with $5 \sec$ trains of $10-20 \mathrm{~Hz}$ stimulation every $30 \mathrm{sec}$ ), the branches and boutons of the presynaptic terminal become highly fluorescent (Fig. $1 B$, data point $b$ in $1 E$ ). This staining is persistent; continuous washing of the preparation for 25 min with control saline caused only minimal loss of fluorescence (Fig. 1C, data point $c$ in $1 E$ ). However, if the nerve was stimulated while washing the preparation, the fluorescence decreased rapidly (Fig. $1 D$, data point $d$ in $1 E$ ). After 15 min of stimulation, fluorescence had decreased by more than half; additional stimulation failed to enhance the extent of destaining (Fig. 1E).

Lizard nerve terminals can also be stained and destained with FM1-43 in the absence of nerve activity (i.e., action potentials) by directly depolarizing the nerve endings with high-K saline (60 mM) in the presence of tetrodotoxin $(1 \mu \mathrm{M})$. The staining and destaining produced by high $\mathrm{K}$ was indistinguishable from that produced by nerve stimulation. Thus, all subsequent staining used direct depolarization with $60 \mathrm{~mm}$ potassium (i.e., high-K saline).

To establish that staining and destaining with FM1-43 was caused by cycles of endocytosis and exocytosis of synaptic vesicles rather than by some other consequence of nerve activity, experiments were performed in which $\mathrm{Cd}^{2+}$ was added to the external bathing solution to block $\mathrm{Ca}^{2+}$ influx and prevent transmitter release (Lindgren and Moore, 1989). Addition of $\mathrm{Cd}^{2+}$ to the saline $\left(\mathrm{Ca}^{2+}\right.$ was also removed from the bathing solution to further reduce the possibility of neurotransmitter release) significantly reduced both the depolarization-dependent FM1-43 uptake and destaining of motor nerve terminals (data not shown).

The amount of FM1-43 staining in the $\mathrm{Cd}^{2+}$ saline was $31 \pm 6 \%$ (mean \pm SEM; $n=5$ ) the amount of staining observed under control conditions in which neurotransmitter release was allowed. This difference was determined to be statistically significant $(p<$ 0.05 , two-tailed $t$ test assuming unequal variances). In preparations already loaded with FM1-43, the extent of destaining in the $\mathrm{Cd}^{2+}$ saline $(n=5)$ was found on average to be $43 \%$ of the amount of destaining measured in the control high-K saline. This difference was also determined to be statistically significant $(p<$ 0.05 , two-tailed $t$ test assuming unequal variances). Similar $\mathrm{Cd}^{2+}$ sensitivity was observed when FM1-43 cycling was evoked by nerve stimulation (data not shown). Therefore, $50-70 \%$ of the staining and destaining of nerve terminals with FM1-43 is calcium-dependent.

\section{Propionate acidifies the inside of lizard motor nerve terminals}

To investigate the $\mathrm{pH}$ dependence of endocytosis, it was first necessary to establish a method for reliably acidifying the interior of the nerve terminals. To detect changes in internal $\mathrm{pH}$, nervemuscle preparations were loaded with the fluorescent $\mathrm{pH}$ indicator BCECF. The replacement of chloride in the external bathing solution with propionate proved to be a reliable method for decreasing the intracellular $\mathrm{pH}$ of the motor nerve terminals. The anions of weak acids, such as propionic acid, are known to acidify the interior of cells (Sharp and Thomas, 1981). Although the anions themselves are relatively impermeable, the weak acids with which they are in equilibrium readily cross cell membranes. As the weak acids diffuse into the cell, they dissociate and release free hydrogen ions, decreasing intracellular $\mathrm{pH}$. An example is shown in Figure $2 A$. In a terminal loaded with BCECF, $100 \mathrm{~mm}$ of the chloride in the external bathing solution was replaced first with acetate and then with propionate for $15 \mathrm{~min}$ each. Note that each weak acid caused a reversible decrease in BCECF fluorescence of similar magnitude. This suggests that propionate and acetate have similar acidifying potency, a result that is consistent with the similarity in their chemical structure and $\mathrm{p} K_{\mathrm{a}}$ values (Sharp and Thomas, 1981). Separate experiments demonstrate that the sustained application of propionate can acidify nerve terminals for up to $2 \mathrm{hr}$ (see Fig. 6).

The concentration dependence of propionate's effect on internal $\mathrm{pH}$ was determined by substituting different concentrations of propionate for chloride in the external saline and measuring changes in the fluorescence of motor nerve terminals loaded with BCECF. The relative change in BCECF fluorescence was determined for each application of propionate by dividing the maximum change in fluorescence $(\Delta F)$ by the fluorescence intensity immediately before the application of propionate $\left(F_{\mathrm{o}}\right)$. A summary of these experiments is presented in Figure $2 B$. Note that a linear relationship exists between the relative change in BCECF fluorescence and the concentration of propionate up to $100 \mathrm{~mm}$. Additional increases in propionate concentration beyond $100 \mathrm{~mm}$ resulted in a relatively smaller incremental change in BCECF fluorescence.

\section{FM1-43 staining, but not destaining, is reduced by internal acidification}

The effect of internal acidification on endocytosis was examined by measuring the uptake of FM1-43 into nerve terminals stimu- 
A.

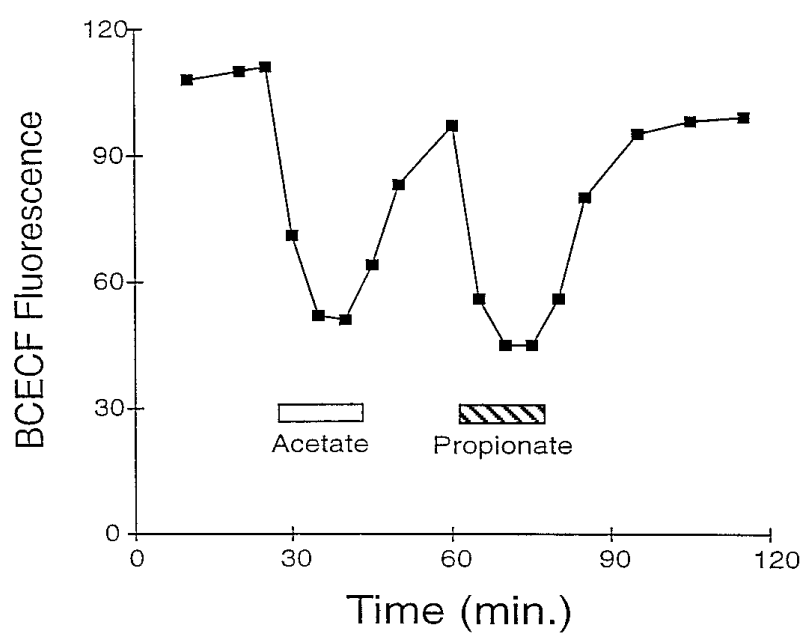

B.

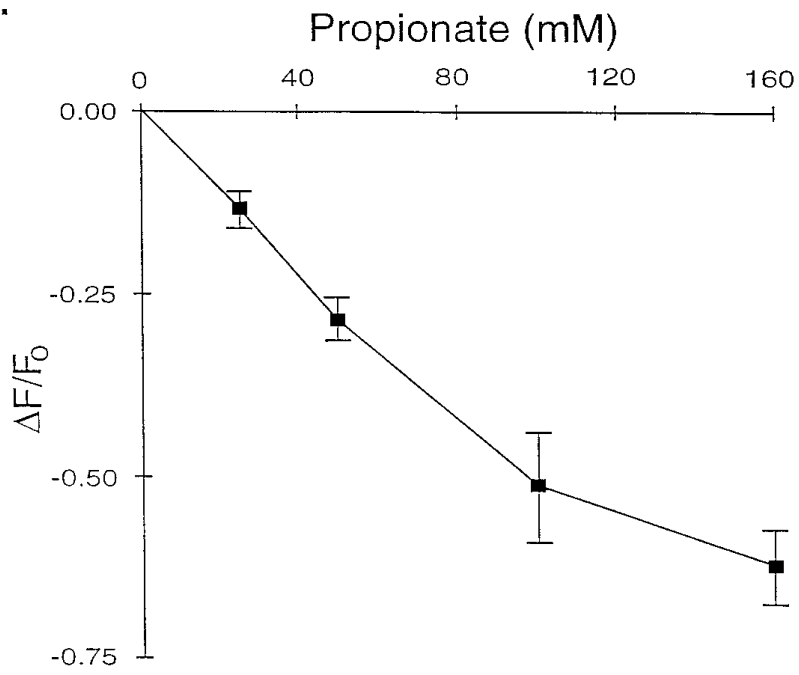

Figure 2. External application of either acetate or propionate acidifies the inside of the nerve terminal. $A$, The $\mathrm{pH}$-sensitive dye BCECF was loaded into a preparation, and the intensity of fluorescence emission from a nerve terminal was quantified with a confocal microscope. (Decreasing fluorescence corresponds to decreasing $\mathrm{pH}$.) Both acetate and propionate were applied at a concentration of $100 \mathrm{~mm}$, where indicated. $B$, Summary of several experiments showing the effect of propionate concentration on the relative change of BCECF fluorescence emission. $\Delta F$ is the maximum change in BCECF fluorescence measured after applying propionate. $F_{0}$ is the amount of fluorescence measured immediately before applying the propionate. Each point represents the mean $\pm \operatorname{SEM}(n=5$ for each point, except $100 \mathrm{~mm}$ propionate, for which $n=10$ ).

lated by high-K saline in the presence of propionate. As shown in Figure $3 A$, relatively little FM1-43 is taken up into terminals stimulated with high-K saline if the intracellular $\mathrm{pH}$ of the nerve terminal is simultaneously lowered by substituting the chloride in the external bathing solution with propionate. A similar reduction in FM1-43 uptake was also seen when propionate was added before as well as during high-K saline application. The reversibility of reduction in FM1-43 uptake is readily demonstrated by removing the propionate (replacing the chloride) and restimulating the nerve with high-K saline in the presence of FM1-43. Under these conditions, the terminal becomes loaded with FM1-43 (Fig. 3A).
Experiments were performed using propionate concentrations of $160,100,50$, and $25 \mathrm{~mm}$ (the remainder of the anion in the external solution was chloride). Neither 25 nor $50 \mathrm{~mm}$ propionate had any effect on FM1-43 uptake (data not shown). However, both 100 and $160 \mathrm{~mm}$ propionate were equally effective at inhibiting FM1-43 uptake. On average, the amount of FM1-43 staining in the presence of at least $100 \mathrm{~mm}$ propionate was $35 \pm 4 \%$ (mean $\pm \mathrm{SEM}, n=8$ ) the amount of staining observed under control conditions.

To control for the possibility that acidification affects the fluorescent intensity of FM1-43 or causes a nonspecific leakage of dye from inside vesicles, stained preparations were incubated in propionate saline with the normal extracellular concentration of $\mathrm{K}^{+}$. Propionate did not change the fluorescent intensity of FM1-43 stained terminals in the absence of depolarization. Thus, the reduced fluorescent staining intensity of FM1-43 in nerve terminals after depolarization in propionate saline is attributable to an action on FM1-43 uptake and not to a curious nonspecific effect of propionate on the indicator itself.

In contrast to the reduced FM1-43 staining during internal acidification, a decrease in intracellular $\mathrm{pH}$ has no effect on the unloading of FM1-43. After the nerve terminal depicted in Figure $3 A$ was successfully loaded with FM1-43, stimulation with high-K saline induced the rapid loss of FM1-43, despite the substitution of chloride in the external bathing solution with propionate. Figure $3 B$ summarizes the results from 10 experiments in which preparations preloaded with FM1-43 were destained by high-K saline in either the presence or the absence of propionate. The mean percentage of stain lost by $15 \mathrm{~min}$ of high-K stimulation was the same under both conditions $(\sim 45 \%)$. Thus, at levels of intracellular acidification where endocytosis is reduced, the extent of exocytosis, as determined by FM1-43 unloading, is unaffected, indicating that propionate selectively affects FM1-43 uptake.

\section{Estimating the amount of acidification required to block endocytosis}

Endocytosis, as monitored by the uptake of FM1-43, was insensitive to changes in internal $\mathrm{pH}$ caused by the application of 25 or $50 \mathrm{~mm}$ propionate. However, application of 100 or $160 \mathrm{~mm}$ propionate maximally reduced FM1-43 uptake. To approximate the actual internal $\mathrm{pH}$ at which endocytosis is arrested, relative changes in BCECF fluorescence were converted to actual $\mathrm{pH}$ changes.

To convert BCECF fluorescence to actual $\mathrm{pH}$ values, it was first necessary to estimate the normal intracellular $\mathrm{pH}$ of the motor nerve terminals. Normal intracellular $\mathrm{pH}$ was estimated to be 7.5 using the nigericin "null-point" method (see Materials and Methods) (Thomas et al., 1979). Using published emission spectra for BCECF (Molecular Probes) and the average change in BCECF fluorescence $\left(\Delta \mathrm{F} / F_{0}\right)$ created by $100 \mathrm{~mm}$ propionate, we determined that FM1-43 loading is reduced if the internal $\mathrm{pH}$ of the nerve terminal decreases from 7.5 to 6.8 . On the other hand, using the average change in BCECF fluorescence $\left(\Delta F / F_{0}\right)$ created by 50 mM propionate, we concluded that FM1-43 loads normally if $\mathrm{pH}$ decreases only to 7.1. Thus, to reduce FM1-43 uptake, internal $\mathrm{pH}$ must decrease by between 0.4 and $0.7 \mathrm{pH}$ units.

\section{The presence of propionate per se in the external saline is not sufficient to block FM1-43 staining}

Although the above results strongly suggest that FM1-43 uptake is inhibited by internal acidification, they are also consistent with a direct $\mathrm{pH}$-independent effect of propionate. As a first step in 


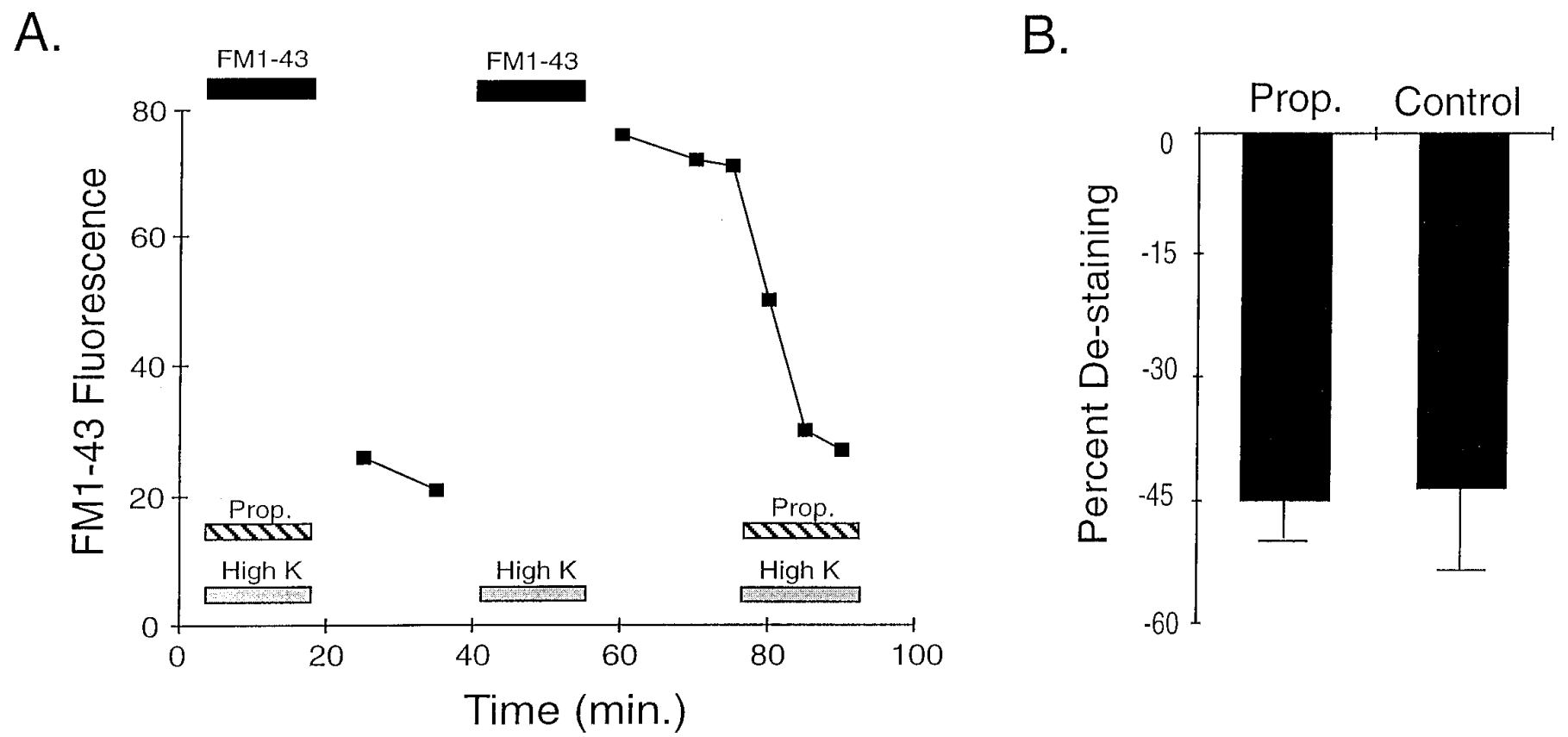

Figure 3. Application of propionate prevents FM1-43 staining but does not prevent destaining of nerve terminals preloaded with FM1-43. $A$, The average intensity of fluorescence emission from a nerve terminal is plotted as a function of time. FM1-43 was applied at $2 \mu \mathrm{M}$ (FM1-43), propionate at $160 \mathrm{~mm}$ (Prop.), and potassium at $60 \mathrm{~mm}$ (High K). B. The amount of FM1-43 stain lost relative to the amount preloaded was determined after the application of high-K saline in either the presence or the absence of propionate. The mean percent destaining \pm SEM in the presence of propionate ( $\geq 100$ $\mathrm{mM}$ ) is represented by the column on the left $(n=5)$. The mean percent destaining \pm SEM in the absence of propionate (control saline) is represented by the column on the right $(n=5)$.

evaluating whether FM1-43 uptake might be pH-sensitive, we substituted $\mathrm{Cl}^{-}$with acetate and found a similar reduction in FM1-43 uptake (data not shown). To further test the role of $\mathrm{pH}$ in controlling dye uptake, conditions were established under which the addition of $100 \mathrm{~mm}$ propionate to the external saline had little effect on internal $\mathrm{pH}$. One way to lower the concentration of propionic acid, independent of changing the total concentration of propionate, involves increasing the $\mathrm{pH}$ of the external solution. At a higher $\mathrm{pH}$, less propionate will be protonated, resulting in a lower concentration of propionic acid.

The effect of raising external $\mathrm{pH}$ from 7.3 to 8.3 on the acidifying potency of propionate is shown in Figure $4 A$. A preparation was loaded with BCECF, and $100 \mathrm{~mm}$ propionate was applied under conditions of normal external $\mathrm{pH}$ (i.e., 7.3). As had been observed previously (Fig. 2), the presence of propionate in the external bathing solution caused BCECF fluorescence to decrease, indicative of a decrease in the internal $\mathrm{pH}$ of the nerve terminal. However, elevation of external $\mathrm{pH}$ to 8.3 , although itself having only a small effect on BCECF fluorescence (a small decrease), prevented the subsequent addition of propionate from acidifying the nerve terminal (Fig. $4 A$ ).

To determine the effect of external propionate on endocytosis, independent of its effect on internal $\mathrm{pH}$, we examined FM1-43 uptake in $100 \mathrm{~mm}$ propionate saline at an external $\mathrm{pH}$ of 8.3 (Fig. $4 B)$. A preparation was first stimulated with high-K saline and exposed to FM1-43 in the presence of $100 \mathrm{~mm}$ propionate $\left(p H_{\mathrm{o}}\right.$ 7.3). The preparation was then washed for $20 \mathrm{~min}$ and reexposed to FM1-43, this time after changing external $\mathrm{pH}$ to 8.3 (propionate was still present). Although propionate blocked FM1-43 uptake when the external $\mathrm{pH}$ was 7.3, FM1-43 uptake was normal when the external $\mathrm{pH}$ was adjusted to 8.3 , despite the maintained presence of propionate. The amount of FM1-43 staining at an external $\mathrm{pH}$ of 7.3 was $38+6 \%$ (mean $\pm \mathrm{SEM}, n=4$ ) of the staining when the external $\mathrm{pH}$ was increased to 8.3. Thus, the mere presence of $100 \mathrm{~mm}$ propionate in the external solution does not preclude FM1-43 uptake. That this uptake of FM1-43 into the nerve terminal is associated with the endocytotic retrieval of membrane into synaptic vesicles is supported by the subsequent observation that the fluorescence decreases only if the preparation is restimulated with high-K saline (see Fig. 4B).

\section{The intracellular presence of propionate per se is not sufficient to block FM1-43 staining}

To test the possibility that propionate per se in the nerve terminal affects FM1-43 uptake independent of acidification, we explored the possibility of reversing the propionate-induced decrease in $\mathrm{pH}$ by adding ammonium chloride (Boron and DeWeer, 1976). As shown in Figure $5 A$, the internal $\mathrm{pH}$ of a terminal acidified by 100 $\mathrm{mm}$ propionate is transiently restored to normal by the addition of $60 \mathrm{~mm}$ ammonium chloride. Because this method restores internal $\mathrm{pH}$ by directly buffering hydrogen ions released inside the nerve terminal (with ammonia), rather than by reducing the diffusion of propionate into the terminal (as propionic acid), it provides a direct test for the $\mathrm{pH}$ sensitivity of FM1-43 uptake.

If intracellular acidification is necessary to block endocytosis, rather than the intracellular presence of propionate, then FM1-43 uptake should be restored when ammonium chloride is applied to a preparation already acidified by propionate. An experiment designed to test this is shown in Figure $5 B$. A preparation was stimulated with high-K saline and exposed to FM1-43 in the presence of $100 \mathrm{~mm}$ propionate (at an external $\mathrm{pH}$ of 7.3). As established previously, only a small amount of FM1-43 is taken up into the terminal under these conditions (see Figs. $3 A, 4 B$ ). The preparation was then washed for $20 \mathrm{~min}$ and reexposed to FM1- 
A.

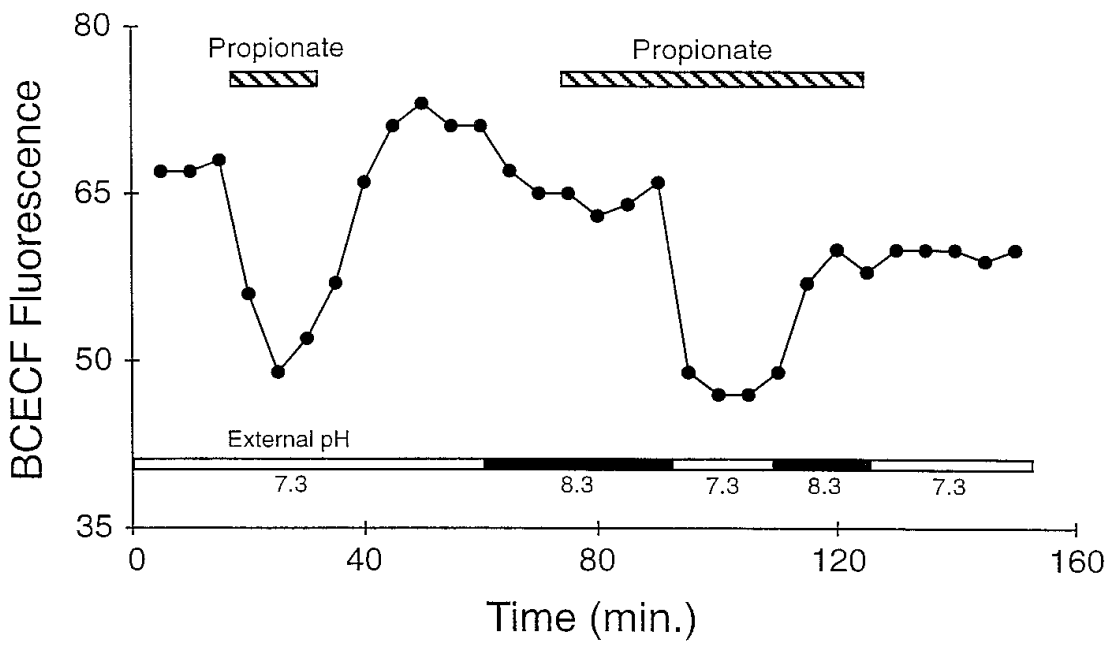

B.

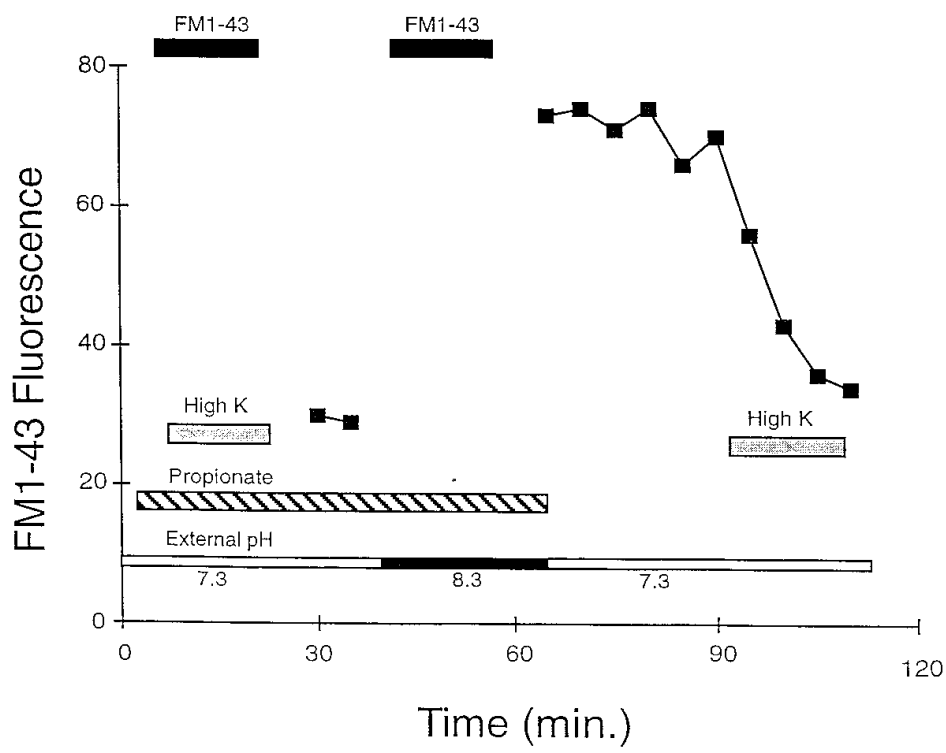

Figure 4. The ability of propionate to acidify the nerve terminal and prevent FM1-43 loading is mitigated by raising external $\mathrm{pH}$. $A, \mathrm{BCECF}$ was loaded into a preparation, and the intensity of fluorescence emission from a nerve terminal was quantified. (Decreasing fluorescence corresponds to decreasing $\mathrm{pH}$.) When external $\mathrm{pH}$ was raised to 8.3 by adding $\mathrm{KOH}$ to the external saline, propionate $(100 \mathrm{~mm})$ no longer acidified the nerve terminal. $B$, The average intensity of fluorescence emission from a nerve terminal was plotted as a function of time. Concentrations are FM1-43, $2 \mu \mathrm{M}$; propionate, $100 \mathrm{~mm}$; potassium (high $\mathrm{K}$ ), $60 \mathrm{~mm}$. Note that FM1-43 loading did not require a second application of high-K saline (for an explanation, see Discussion).
43, this time after adding $60 \mathrm{~mm}$ ammonium chloride $(100 \mathrm{~mm}$ propionate was still present). Despite the maintained presence of propionate in the external saline (at a $p H_{\mathrm{o}}$ of 7.3), FM1-43 was taken up into the nerve terminal when it was applied along with ammonium chloride. Most of the FM1-43 taken up under these conditions could be unloaded from the nerve terminal by stimulating with high-K saline. Taken together, these data are consistent with propionic acid interfering with FM1-43 uptake through intracellular acidification.

\section{Intracellular acidification can delay FM1-43 uptake for up to 2 hr}

In studies of the nerve terminal, it would be of great experimental advantage to be able to temporally uncouple exocytosis from endocytosis. Toward this goal, we determined whether the sustained presence of propionate causes a maintained acidification of the nerve terminal. Figure $6 A$ shows that application of propionate for up to $2 \mathrm{hr}$ does acidify the nerve terminal for the complete period of addition of this weak acid. To determine whether the activity-dependent uptake of FM1-43 was delayed for this time period, we depolarized nerve terminals in high-K saline in the presence of propionate, then maintained the preparation in propionate for $2 \mathrm{hr}$. At washout of propionate, we added FM1-43 and asked whether this fluorescent indicator now stained the nerve terminal. Figure $6 \mathrm{~B}$ clearly demonstrates that the activitydependent uptake of FM1-43 is delayed by the presence of propionate in the bathing saline for this $2 \mathrm{hr}$ period.

\section{Internal acidification of the nerve terminal prevents the recycling of synaptic vesicles}

The experiments described above demonstrate that the uptake of FM1-43 into motor nerve terminals is significantly reduced by internal acidification. This suggests that endocytosis or, more specifically, that the endocytotic movement of membrane from the plasma membrane to synaptic vesicles is sensitive to decreases in $\mathrm{pH}$. To test this latter suggestion more directly, the recycling of synaptic vesicles was studied via electron microscopy. A nervemuscle preparation was stimulated with high-K saline containing $100 \mathrm{~mm}$ propionate for $20 \mathrm{~min}$ and was then immediately fixed for electron microscopy. An example of a nerve terminal from this 
A.

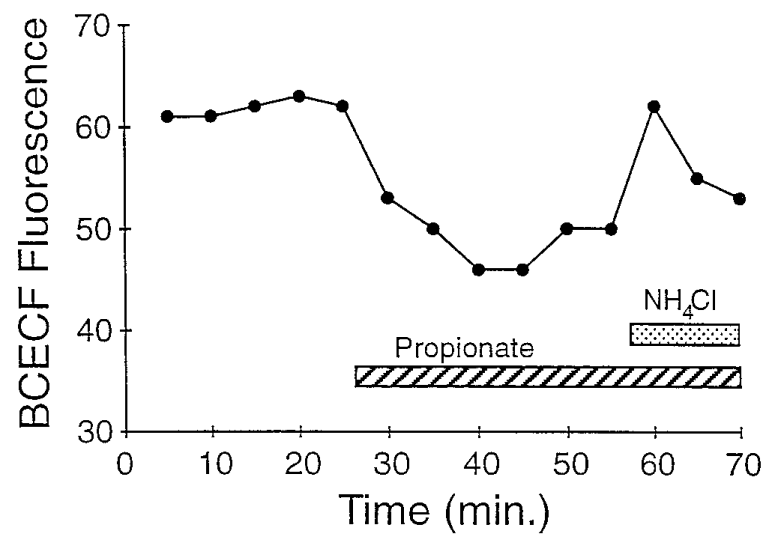

B.

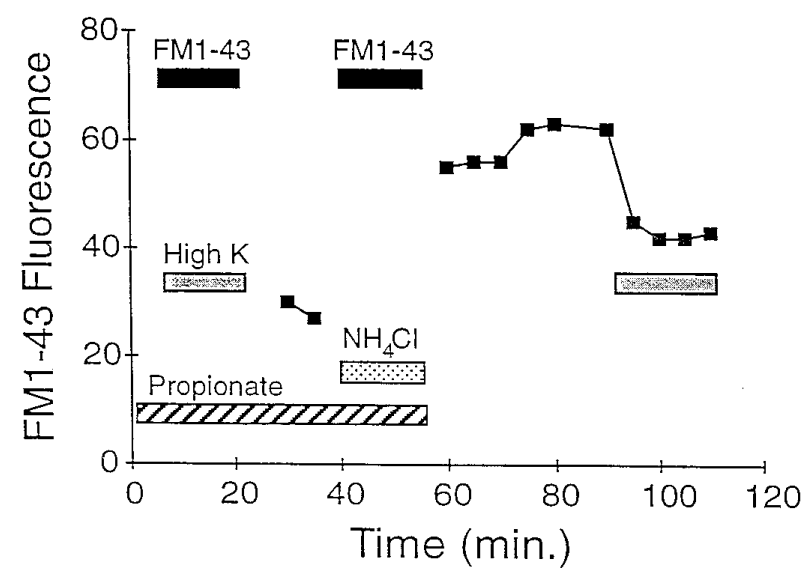

Figure 5. Ammonium chloride transiently reverses the acidification induced by propionate and restores FM1-43 loading. $A$, BCECF was loaded into a preparation, and the intensity of fluorescence emission from a nerve terminal was quantified. The application of ammonium chloride transiently reverses the internal acidification induced by propionate. $B$, The average intensity of fluorescence emission from a nerve terminal is plotted as a function of time. Endocytosis, which has been blocked by internal acidification, is restored by the application of ammonium chloride. Note that FM1-43 loading did not require a second application of high-K saline (for an explanation, see Discussion). Concentrations are FM1-43, $2 \mu \mathrm{M}$; propionate, $100 \mathrm{~mm}$; ammonium chloride $\left(\mathrm{NH}_{4} \mathrm{Cl}\right), 60 \mathrm{~mm}$; potassium (high K), $60 \mathrm{~mm}$.

preparation is shown in Figure $7 B$. In contrast to the nerve terminal shown in Figure $7 A$, which is in an unstimulated control preparation, and to Figure $8 B$, which is a preparation stimulated in high-K saline and allowed to recover for $20 \mathrm{~min}$ in normal saline, the terminal shown in Figure $7 B$ portrays a conspicuous paucity of synaptic vesicles. In addition to containing fewer synaptic vesicles, the cytoplasm is punctuated with numerous loops and swirls of membrane.

These morphological observations are consistent with the measurements of FM1-43 uptake, which suggested that acidification of the nerve terminal reduces the internalization of plasma membrane via endocytosis. More specifically, the disappearance of synaptic vesicles revealed in Figure $7 B$ suggests that internal acidification blocks, or significantly retards, the recycling of synaptic vesicles. To help confirm the specificity of this effect of internal acidification on synaptic vesicle recycling, the following experiment was performed. The neuromuscular junctions in two different muscles were first depleted of synaptic vesicles by stimulating with high-K saline containing $100 \mathrm{~mm}$ propionate for 20 $\min$. (Note that this is the same treatment applied to the neuromuscular junction shown in Fig. 7B.) Both muscles were then allowed to recover for $20 \mathrm{~min}$ in saline with normal $\mathrm{K}(3 \mathrm{~mm})$ and fixed immediately for electron microscopy. One of the muscles was kept in the presence of propionate $(100 \mathrm{~mm})$ throughout the recovery, and the other was allowed to recover in normal saline.

As shown in Figure $8 A$, the nerve terminals in the muscle that recovered in the presence of propionate are still relatively devoid of synaptic vesicles and contain loops and swirls of membrane. Figure $8 C$ shows a high magnification of a portion of a nerve terminal from this preparation in which the loops of membrane are clearly seen in continuity with the plasma membrane. In contrast, the nerve terminals in the muscle allowed to recover in normal saline are packed full of synaptic vesicles (Fig. $8 B$ ). Moreover, loops and swirls of membrane are observed infrequently in this condition. Another notable characteristic of terminals that have been permitted to recover from the $\mathrm{pH}$-dependent block of endocytosis is that they possess many coated structures. An example is shown in Figure $8 D$, in which a small portion of a nerve terminal allowed to recover in normal saline is shown to contain several coated vesicles and at least one structure that appears to be a coated vesicle forming at the end of a loop of membrane.

\section{DISCUSSION}

\section{Internal acidification arrests endocytosis associated with synaptic vesicle recycling}

The principal finding reported in this paper is that internal acidification of a motor nerve terminal reduces endocytosis required in the recycling of synaptic vesicles. If the internal $\mathrm{pH}$ of the nerve terminal is decreased by replacing at least $100 \mathrm{~mm}$ of the chloride in the external bathing solution with propionate, the activitydependent uptake of FM1-43 is reduced. (The activity-dependent unloading of FM1-43 is unaffected.) The uptake of FM1-43 is restored if propionate is removed from the external bathing solution or if propionate's efficacy at decreasing intracellular $\mathrm{pH}$ is mitigated by raising external $\mathrm{pH}$ or by co-applying ammonium chloride. These results, together with the electron microscopic demonstration that the recycling of synaptic vesicles after high-K stimulation is compromised by exposure to $100 \mathrm{~mm}$ propionate, suggest that intracellular acidification impairs endocytosis in motor nerve terminals.

In addition to supporting the principal conclusions of this study, the electron micrographs suggest some intriguing possibilities regarding the mechanism by which internal acidification inhibits synaptic vesicle recycling. As reported previously by Ceccarelli et al. (1989) and Gennaro et al. (1978), the inhibition of synaptic vesicle recycling is associated with the appearance of numerous, long swirls of membrane in the cytoplasm of the nerve terminal. In a few cases, these swirls are observed to be continuous with the presynaptic membrane (see Fig. $8 C$ ), which suggests that they represent deep invaginations of the plasma membrane. Because these swirls of membrane are rarely seen in normal nerve terminals, they are probably a pathological response of the nerve terminal to the inhibition of endocytosis. Nevertheless, their production is consistent with endocytosis being stopped at a relatively 
A.

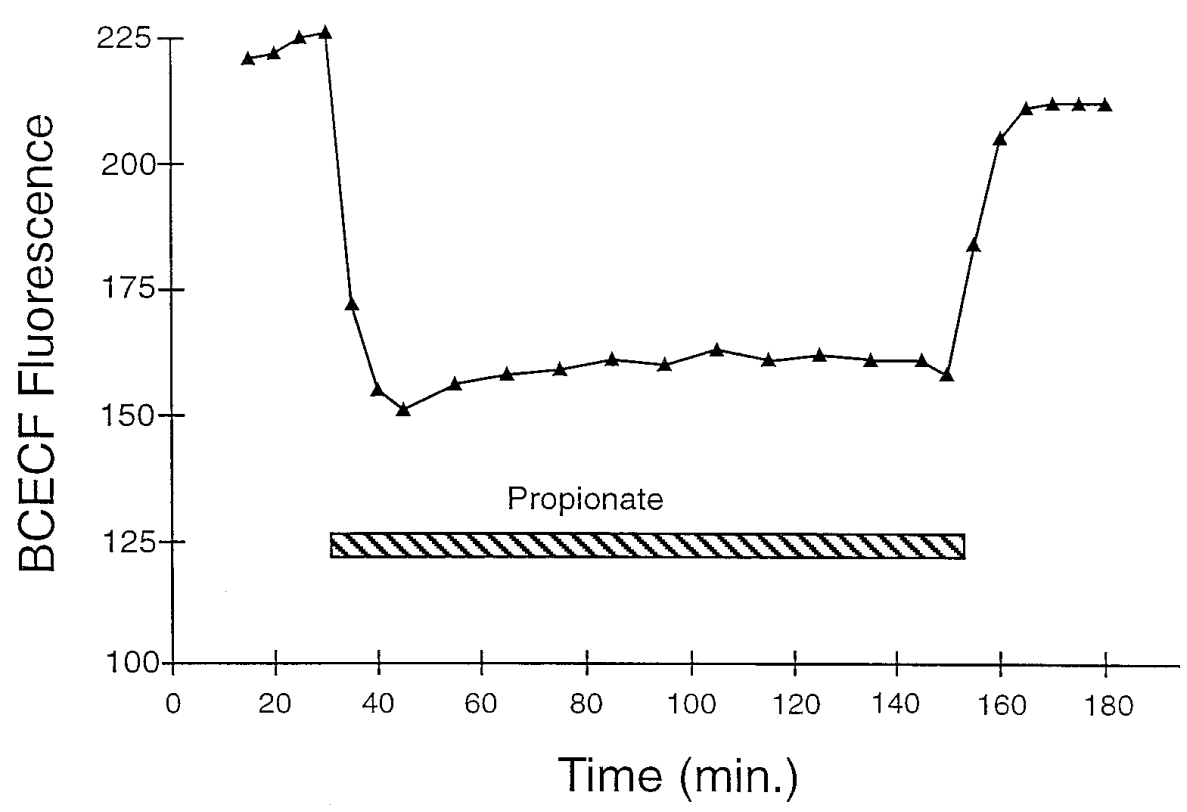

B.

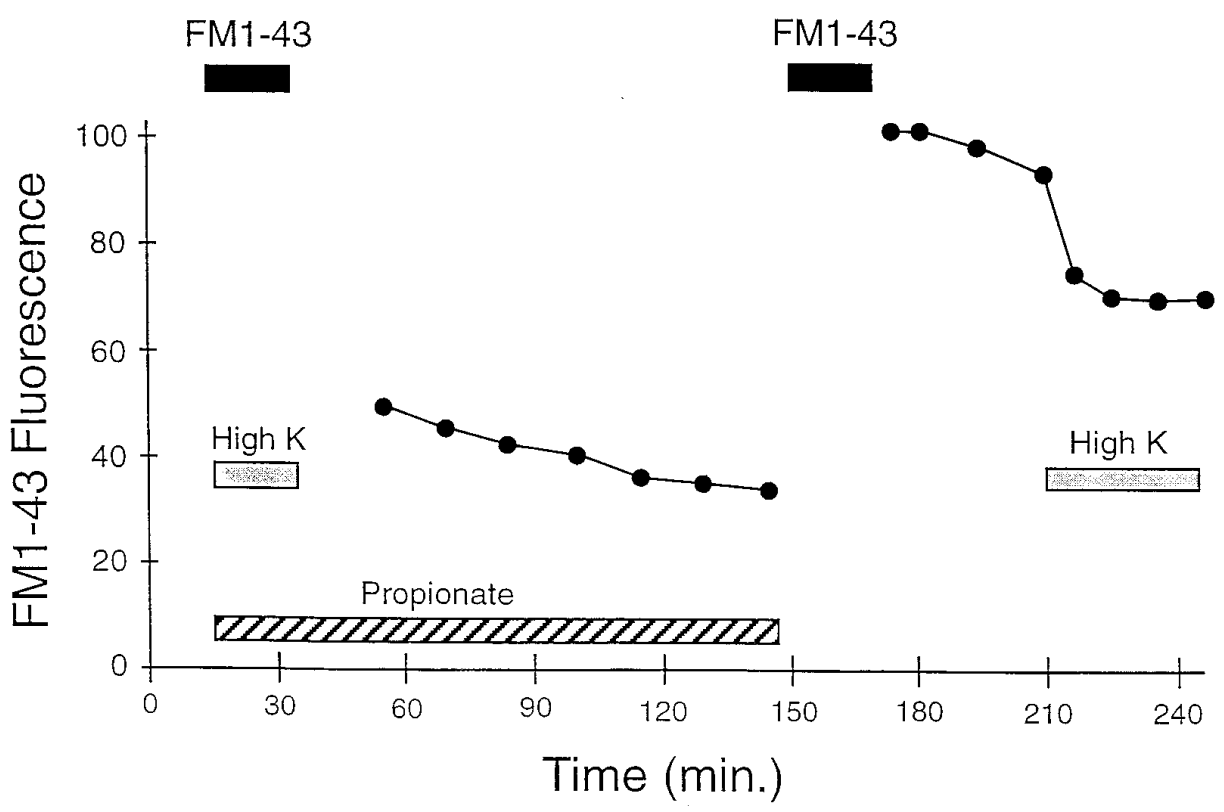

Figure 6. Continuous application of propionate acidifies the nerve terminal and reduces endocytosis for at least $2 \mathrm{hr}$. $A$, BCECF was loaded into a preparation, and the intensity of fluorescence emission from a nerve terminal was quantified. The internal $\mathrm{pH}$ of the nerve terminal was acidified throughout a $2 \mathrm{hr}$ application of propionate. $B$, The average intensity of fluorescence emission from a nerve terminal is plotted as a function of time. Endocytosis was restored immediately after removing propionate, which had been applied to the preparation for longer than 2 hr. Note that FM1-43 loading did not require a second application of high-K saline (for an explanation, see Discussion). Concentrations are FM1-43, $2 \mu \mathrm{M}$; propionate, $100 \mathrm{~mm}$; potassium (high $\mathrm{K}$ ), $60 \mathrm{mM}$.

late stage in the process, such as the fission step where the invaginating coated pit is separated from the presynaptic membrane. Such an interpretation is also consistent with the observation that terminals allowed to recover from vesicle depletion by incubation in normal saline contain many coated structures (coated pits and vesicles) clustered near the closed ends of the invaginating loops of membrane (see Fig. 8D).

\section{Studying membrane recycling in lizard motor nerve terminals}

FM1-43 uptake into lizard motor nerve terminals is qualitatively similar to its uptake into motor nerve terminals of the frog (Betz et al., 1992) and rat (Ribchester et al., 1994). Interestingly, the amount of FM1-43 taken up into lizard nerve endings in the absence of neurotransmitter release (i.e., in the presence of $\mathrm{Cd}^{2+}$ ) was approximately one third of that induced by $15 \mathrm{~min}$ of stimulation with high-K saline. This is somewhat larger than what has been reported at the frog neuromuscular junction but is similar to mammalian neuromuscular junctions (Ribchester et al., 1994). A similar proportion of uptake was also observed when endocytosis was arrested by internal acidification, raising the possibility that the same fraction of FM1-43 uptake that is insensitive to $\mathrm{Cd}^{2+}$ is also insensitive to acidification .

The destaining of lizard motor nerve terminals preloaded with FM1-43 is also qualitatively similar to the destaining of frog nerve 


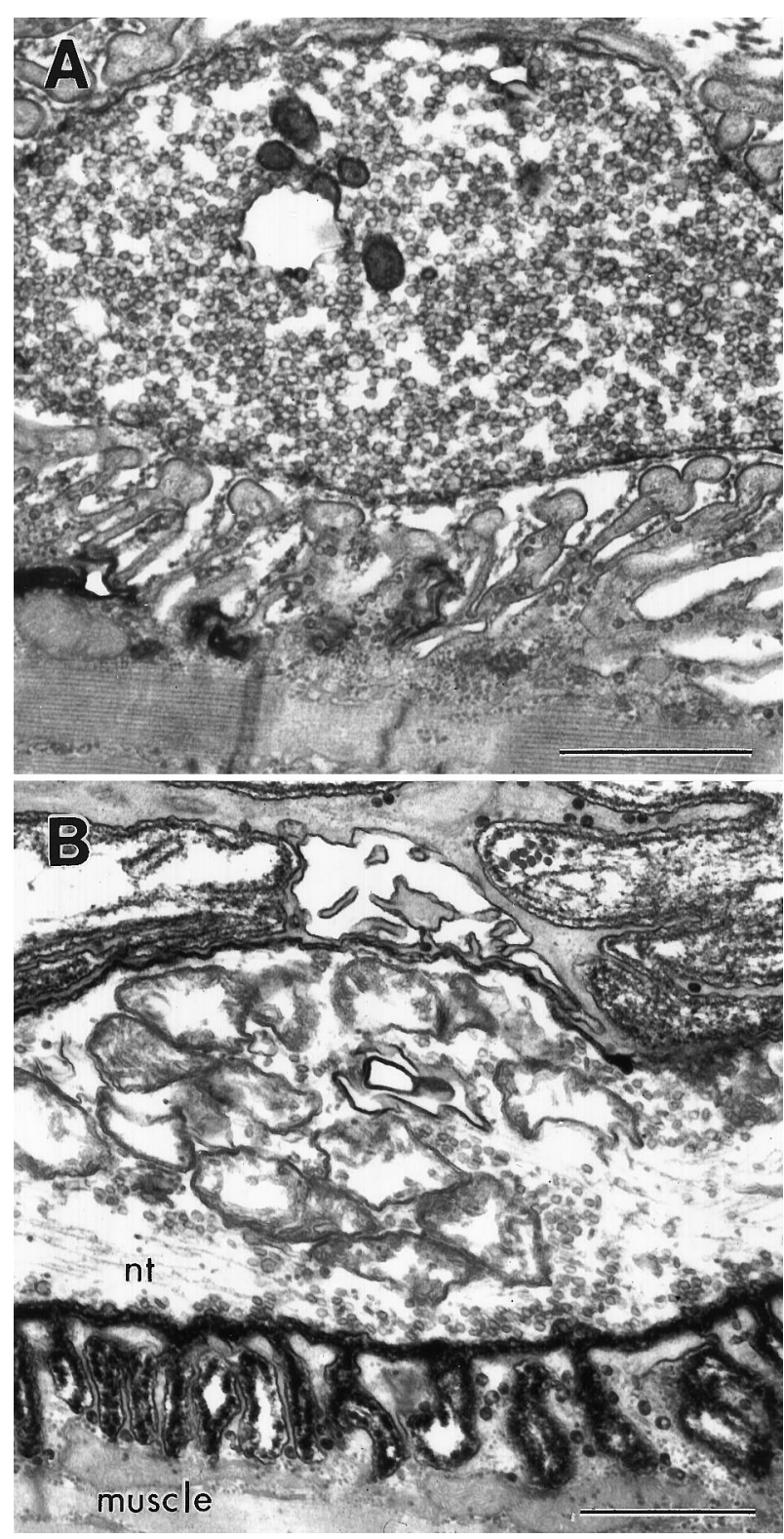

Figure 7. High-K stimulation depletes nerve terminals of synaptic vesicles under conditions of intracellular acidification. Electron micrographs of longitudinal sections from two different neuromuscular junctions. $A$, Resting preparation incubated in control saline before fixation. $B$, Preparation fixed after $20 \mathrm{~min}$ incubation in saline containing propionate (100 $\mathrm{mm})$ and high $\mathrm{K}(60 \mathrm{~mm})$. Note that in $B$, there are fewer synaptic vesicles in the nerve terminal $(n t)$, and the cytoplasm contains swirls and loops of membrane. Scale bars, $1 \mu \mathrm{m}$.

terminals (Betz et al., 1992). However, lizard nerve terminals never lose all of the FM1-43 stain, despite continuous stimulation. On average, only half of the FM1-43 taken up into lizard nerve terminals can be destained by neurotransmitter release (see Fig. $3 B$ ). Residual fluorescence was also reported in mammalian motor nerve terminals (Ribchester et al., 1994) and was attributed to phototoxic damage, because similar residual staining could be observed if frog neuromuscular junctions were overilluminated (Betz et al., 1992). Another possibility is that FM1-43 labels an internal membrane compartment in lizard and rat nerve terminals that is not releasable, either because it is not in synaptic vesicles or it is in a population of synaptic vesicles with very low probability of release (the latter suggestion was made by Ribchester et al., 1994). Additional work is need to distinguish between these possibilities. Nonetheless, $\sim 50-70 \%$ of the FM1-43 staining present is in the form of a pool that is both activity- and calcium-dependent.

To interpret data when using propionate, it is critical that the resulting acidification selectivity act on endocytosis. Because acidification might generally affect cellular metabolism, endocytosis could be reduced as a result of nonspecific metabolic change. However, this is highly unlikely, because acidification does not reduce depolarization-dependent destaining of FM1-43 from nerve terminals. Additionally, after $2 \mathrm{hr}$ of acidification, nerve terminals can take up FM1-43 immediately on removal of propionate, indicating a normal metabolic state of the nerve terminal.

Because propionate reduces the ability of frog (Ceccarelli et al., 1989) and lizard neuromuscular junctions to recycle synaptic vesicles, it will be important to determine the generality of this connection between $\mathrm{pH}$ and endocytosis in nerve terminals. Although intracellular acidification has been shown to inhibit endocytosis in certain non-neuronal cells, such as baby hamster kidney cells (Davoust et al., 1987), human cell lines (Sandvig et al., 1987), and cultured chicken fibroblasts (Heuser, 1989), the rapid retrieval of membrane after exocytosis is reportedly insensitive to low cytosolic $\mathrm{pH}$ in neuroendocrine cells, such as rat melanotrophs (Thomas et al., 1994) and chromaffin cells (Artalejo et al., 1995; Burgoyne, 1995). It remains to be determined whether fast central synapses exhibit $\mathrm{pH}$-sensitive endocytosis.

It is interesting to speculate that the sensitivity of endocytosis to $\mathrm{pH}$ in some cells and apparent lack of sensitivity in others may be a function of the specific molecules participating in endocytosis. For example, internal acidification may block endocytosis only if it is mediated by clathrin. Thomas et al. (1994), Burgoyne (1995), and Artalejo et al. (1995) report that the $\mathrm{pH}$-insensitive form of endocytosis that they monitored is not mediated by a clathrindependent mechanism. Because we observed coated vesicles in the lizard neuromuscular junction (Fig. 8D) and endocytosis is sensitive to $\mathrm{pH}$, it is likely that clathrin-mediated endocytosis is functional in these motor nerve terminals.

The $\mathrm{pH}$-induced delay of endocytosis after exocytosis may permit insight into the mechanisms and requirements of endocytosis in a nerve terminal. The normal close coupling between exocytosis and endocytosis has made it difficult to elucidate the requirements of endocytosis per se (for an example, see Artalejo et al., 1996). However, the ability of intracellular acidification to temporally uncouple exocytosis from endocytosis may afford an opportunity to untangle the requirements for endocytosis from those for exocytosis.

Although calcium plays a key role in triggering exocytosis, its role in endocytosis is less clear (for an example, see Artalejo et al., 1996) (von-Gersdorff and Matthews, 1994; Wu and Betz, 1996). Because endocytosis is reduced by intracellular acidification (as assayed by electron microscopy and FM 1-43), but proceeds when $\mathrm{pH}$ is restored to physiological levels without additional depolarization of the nerve terminal, we are able to conclude that elevated internal calcium is not required for endocytosis per se. Whether calcium plays a role in regulating initial events that permit endocytosis, however, is not addressed with this approach.

\section{Using BCECF fluorescence to detect changes in intracellular $\mathrm{pH}$}

The above conclusions drawn from the results presented in this paper are based on the assumption that changes in the intensity 

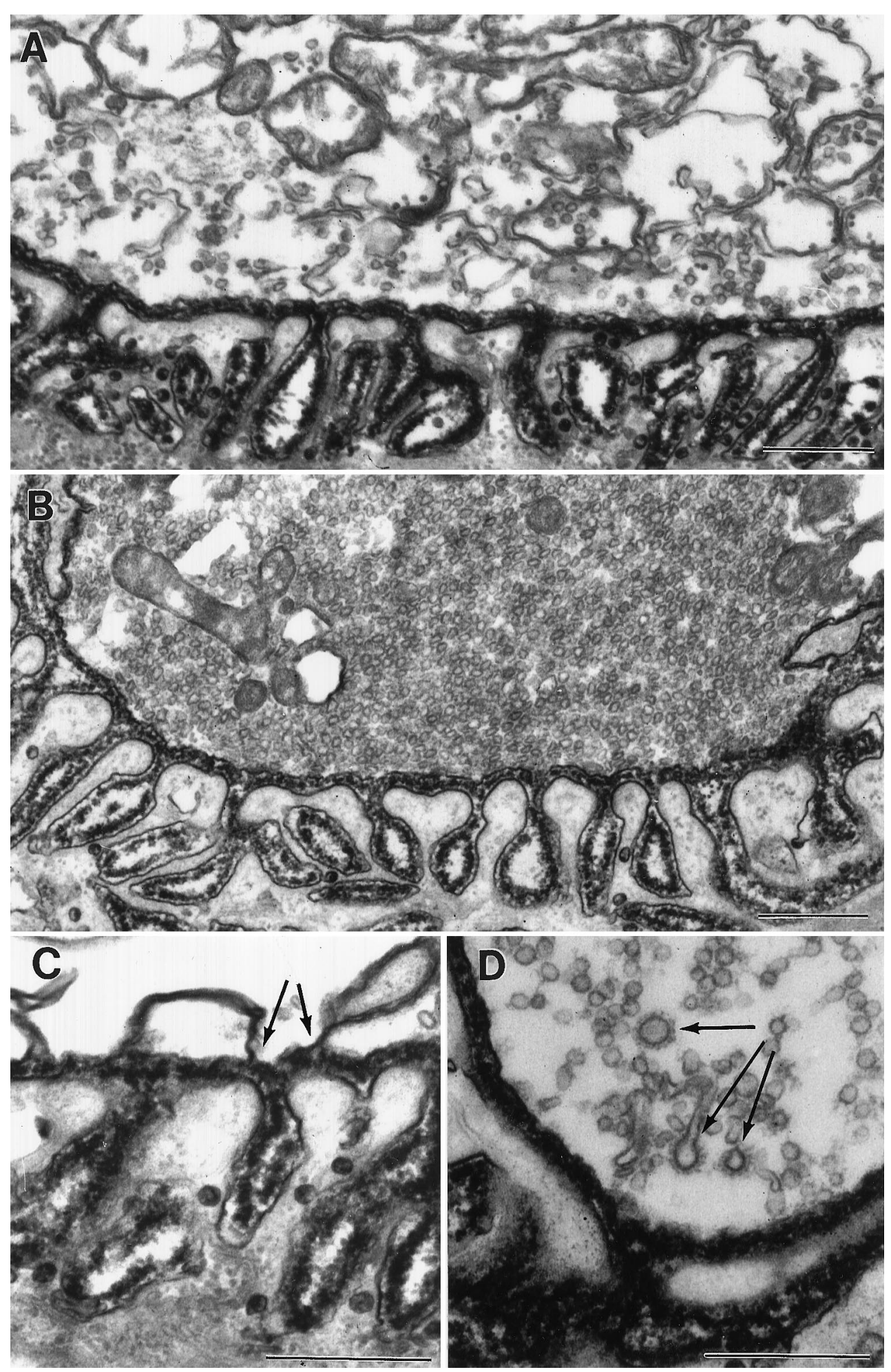

Figure 8. Nerve terminals do not recover from high- $\mathrm{K}$ stimulation if propionate is present. Electron micrographs of longitudinal sections from four different neuromuscular junctions. Preparations were incubated in saline containing propionate (100 mM) and high K (60 mM) for $20 \mathrm{~min}$ to deplete terminals of synaptic vesicles. Preparations were then allowed to recover in normal $\mathrm{K}(3 \mathrm{mM})$ for $20 \mathrm{~min}$ either in saline containing $100 \mathrm{~mm}$ propionate $(A$, $C)$ or in control saline $(B, D)$. In $A$, the nerve terminal is still relatively devoid of synaptic vesicles and contains several loops and swirls of membrane, whereas in $B$, the terminal has refilled with synaptic vesicles. $C$ shows a portion of a terminal where loops of membrane are clearly seen in continuity with the presynaptic membrane (arrows). $D$ shows a portion of a nerve terminal containing several coated structures (arrows). Scale bars, $0.5 \mu \mathrm{m}$. of light emitted from BCECF (>515 nm) after excitation with a single wavelength of light $(488 \mathrm{~nm})$ are caused by changes in the intracellular $\mathrm{pH}$ of the nerve terminals. However, the leakage of BCECF from the cell and photobleaching could also cause the light emission to change. Yet, neither dye leakage nor photobleaching could account for the rapid and reversible changes in BCECF fluorescence induced by propionate (e.g., see Figs. $2 A, 4 A$ ).

A third possibility is that propionate causes cell swelling, result- ing in a rapid and reversible dilution of the dye. Although the volume of the nerve terminal may have increased during the application of propionate and decreased on its removal, the magnitude of this change would have had to have been as much as $50 \%$ of the original volume of the nerve terminal to account for the measured changes in BCECF fluorescence. Although small changes in volume cannot be excluded, this unknown factor could account for only a small percentage of the measured changes in fluorescence. Furthermore, a propionate-induced volume change 
cannot account for the increase in fluorescence associated with the application of ammonium chloride (see Fig. $5 \mathrm{~A}$ ).

In conclusion, this study demonstrates that acidification of the lizard motor nerve terminal reversibly and selectively reduces activity-dependent endocytosis of synaptic vesicles. The ability to block endocytosis without impairing exocytosis should significantly facilitate the study of the cellular and molecular details of endocytosis.

\section{REFERENCES}

Artalejo CR, Henley JR, McNiven MA, Palfrey HC (1995) Rapid endocytosis coupled to exocytosis in adrenal chromaffin cells involves $\mathrm{Ca}^{2+}$ GTP, and dynamin but not clathrin. Proc Natl Acad Sci USA 92:8328-8332.

Artalejo CR, Elhamdani A, Palfrey HC (1996) Calmodulin is the divalent cation receptor for rapid endocytosis, but not exocytosis, in adrenal chromaffin cells. Neuron 16:195-205.

Betz WJ, Bewick GS (1992) Optical analysis of synaptic vesicle recycling at the frog neuromuscular junction. Science 255:200-203.

Betz WJ, Mao F, Bewick GS (1992) Activity-dependent fluorescent staining and destaining of living vertebrate motor nerve terminals. J Neurosci 12:363-375.

Boron WF, DeWeer P (1976) Intracellular pH transients in squid giant axons caused by $\mathrm{CO}_{2}, \mathrm{NH}_{3}$, and metabolic inhibitors. $\mathrm{J}$ Gen Physiol 67:91-112.

Burgoyne RD (1995) Fast exocytosis and endocytosis triggered by depolarisation in single adrenal chromaffin cells before rapid $\mathrm{Ca} 2+$ current run-down. Pflügers Arch 430:213-219.

Ceccarelli B, Molenaar PC, Oen BS, Polak RL, Torri-Tarelli F, van Kempen GTH (1989) The effect of anions on bound acetylcholine in frog sartorius muscle. J Physiol (Lond) 408:233-249.

Chen MS, Obar RA, Schroeder CC, Austin TW, Poodry CA, Wadsworth SC, Vallee RB (1991) Multiple forms of dynamin are encoded by Shibire, a Drosophila gene involved in endocytosis. Nature 351:583-586.

Davoust J, Gruenberg J, Howell KE (1987) Two threshold values of low pH block endocytosis at different stages. EMBO J 6:3601-3609.

De Camilli P, Takei K (1996) Molecular mechanisms in synaptic vesicle endocytosis and recycling. Neuron 16:481-486.

Florey E, Kriebel ME (1988) Reversible effect of depolarization by $\mathrm{K}$-propionate on sub-miniature endplate potential to bell-miniature endplate potential ratios, on miniature endplate potential frequencies and amplitudes, and on synaptic vesicle diameters and densities in frog neuromuscular junctions. Neuroscience 27:1055-1072.
Gennaro JF, Nastuk WL, Rutherford DT (1978) Reversible depletion of synaptic vesicles induced by application of high external potassium to the frog neuromuscular junction. J Physiol (Lond) 280:237-247.

Heuser J (1989) Effects of cytoplasmic acidification on clathrin lattice morphology. J Cell Biol 108:401-411.

Lindgren CA, Moore JW (1989) Identification of ionic currents at presynaptic nerve endings of the lizard. J Physiol (Lond) 414:201-222.

Lindgren CA, Emery DG, Haydon PG (1996) Intracellular acidification arrests endocytosis at the neuromuscular junction. Soc Neurosci Abstr 21:309.15.

Miller TM, Heuser JE (1984) Endocytosis of synaptic vesicle membrane at the frog neuromuscular junction. J Cell Biol 98:685-698.

Ribchester RR, Mao F, Betz WJ (1994) Optical measurements of activity-dependent membrane recycling in motor nerve terminals of mammalian skeletal muscle. Proc R Soc Lond [Biol] 255:61-66.

Sandvig K, Olsnes S, Petersen OW, van Deurs B (1987) Acidification of the cytosol inhibits endocytosis from coated pits. J Cell Biol 105:679-689.

Sharp AP, Thomas RC (1981) The effects of chloride substitution on intracellular $\mathrm{pH}$ in crab muscle. J Physiol (Lond) 312:71-80.

Takei K, McPherson PS, Schmid SL, De Camilli PD (1995) Tubular membrane invaginations coated by dynamin rings are induced by GTP- $\gamma \mathrm{S}$ in nerve terminals. Nature 374:186-190.

Takei K, Mundigl O, Daniell L, De Camilli P (1996) The synaptic vesicle cycle: a single vesicle budding step involving clathrin and dynamin. J Cell Biol 133:1237-1250.

Thomas JA, Buschbaum RN, Zimniak A, Racker E (1979) Intracellular $\mathrm{pH}$ measurements in Ehrlich ascites tumor cells utilizing spectroscopic probes generated in situ. Biochemistry 18:2210-2218.

Thomas P, Lee AK, Wong JG, Almers W (1994) A triggered mechanism retrieves membrane in seconds after $\mathrm{Ca}^{2+}$-stimulated exocytosis in single pituitary cells. J Cell Biol 124:667-675.

van der Bliek AM, Meyerowitz EM (1991) Dynamin-like protein encoded by the Drosophila shibire gene associated with vesicular traffic. Nature 351:411-414.

von-Gersdorff H, Matthews G (1994) Dynamics of synaptic vesicle fusion and membrane retrieval in synaptic terminals [see comments]. Nature 367:735-739.

Wang L, Sudhof TC, Anderson RGW (1995) The appendage domain of $\alpha$-adaptin is a high affinity binding site for dynamin. J Biol Chem 270:10079-10083.

Wu L-G, Betz WJ (1996) Nerve activity but not intracellular calcium determines the time course of endocytosis at the frog neuromuscular junction. Neuron 17:769-779. 